\title{
Novel Partitioning Algorithm for a Gaussian Inverse Wishart PHD Filter for Extended Target Tracking
}

\author{
Peng $\mathrm{Li}^{1,2}$, Hongwei Ge $\mathrm{G}^{1,2, *}$ and Jinlong Yang ${ }^{1,2}$ \\ ${ }^{1}$ Key Laboratory of Advanced Process Control for Light Industry (Jiangnan University), Ministry of Education, \\ Wuxi, 214122, China \\ [e-mail: lipengjiangnan@163.com] \\ ${ }^{2}$ School of Internet of Things Engineering, Jiangnan University, Wuxi, 214122, China \\ [e-mail: ghw8601@163.com] \\ *Corresponding author: Hongwei Ge
}

Received April 3, 2017; revised June 18, 2017; revised July 27, 2017; accepted August 3, 2017;

published November 30, 2017

\begin{abstract}
Use of the Gaussian inverse Wishart PHD (GIW-PHD) filter has demonstrated promise as an approach to track an unknown number of extended targets. However, the partitioning approaches used in the GIW-PHD filter, such as distance partition with sub-partition (DP-SP), prediction partition (PP) and expectation maximization partition (EMP), fails to provided accurate partition results when targets are spaced closely together and performing maneuvers. In order to improve the performance of a GIW-PHD filter, this paper presents a cooperation partitioning (CP) algorithm to solve the partitioning issue when targets are spaced closely together. In the GIW-PHD filter, the DP-SP is insensitive to target maneuvers but sensitive to the differences in target sizes, while EMP is the opposite. The proposed CP algorithm is a fusion approach of DP-SP and EMP, which employs EMP as a sub-partition approach after DP. Therefore, the CP algorithm will be sensitive to neither target maneuvers nor differences in target sizes. The simulation results show that the use of the proposed CP algorithm will improve the performance of the GIW-PHD filter when targets are spaced closely together.
\end{abstract}

Keywords: Cooperation partitioning, CP, GIW-PHD filter, extended target, tracking

This paper is supported by the National Natural Science Foundation of China (No. 61305017), the Graduate Innovation Foundation of Jiangsu Province (No. KYLX16_0782), the 111 Project under Grant No. B12018, and PAPD of Jiangsu Higher Education Institutions. 


\section{Introduction}

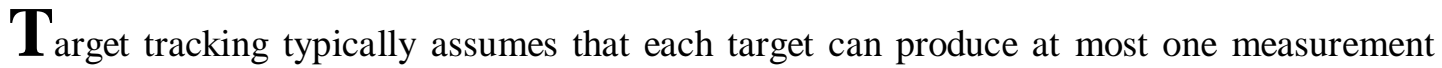
each scan. However, in a modern high-precision sensor system, a target may produce more than a single measurement per scan, such a target called an extended target, and the typical multiple target tracking (MTT) approaches [1-3] fail to track the extended targets accuratly. Therefore, a growing number of works have been done for extended target tracking (ETT) [4-9] in resent years, and this is espacially true for multiple extended target tracking (METT) [10-17].

With finite set statistics (FISST), Mahler presented a random finite set (RFS) based filtering framework [1] to tracking of an unknown number targets. The first order moment of an RFS is the probability hypothesis density (PHD), which is an intensity function defined over the target state space. An important implementation of a MTT PHD filter is the Gaussian mixture PHD (GM-PHD) filter [2], which employs the Gaussian mixture method to approximate the target PHD. The discussion of the newborn target intensity of a GM-PHD can be found in [3].

In ETT, each target may produce a few measurements, thus an ETT filter ought to estimate the target extended state. Koch presented an ETT filter using random matrix (RM) [4], in which the target extended state is defined as a symmetric positive definite random matrix (i.e. covariance matrix). The random matrix is assumed to follow the Wishart distribution, thus the target extended state can be estimated by the inverse Wishart distribution. More discussions about the RM approaches can be found in [5-6]. Another important ETT approach is the random hypersurface models (RHMs) [7-9], which assumes that the measurements are randomly selected from a hypersurface defined by a shape function. A RHM approach can track a target using a Bayesian framework, and meanwhile estimate the extended state (i.e. the target shape) by estimating the parameters of the shape function.

The RM and RHMs approaches cannot track an unknown number of extended targets as well as the typical PHD filters. Therefore, Mahler presented a multiple extended target PHD filter framework [10] for METT. Then, Granström el al. presented a Gaussian mixture implementation of this framework called extended target GM-PHD (ET-GM-PHD) filter [11]. The ET-GM-PHD filter uses the distance partitioning (DP) approach with different distance thresholds to provide several measurement partitions, and then the GM components will be updated by the partition with the largest likelihood. However, when targets are spaced closely together, the DP approach fails to provide accurate partitions, which lead to the estimation error of the ET-GM-PHD filter. To solve this problem, the DP-SP approach was presented in [12]. This approach first employs DP to divide the measurement set into cells, and then uses a so-called sub-partitioning (SP) method to divide the measurement cells produced by closely spaced targets into sub-cells. However, the ET-GM-PHD filter cannot estimate the target extensions (i.e. the target extended states are given values), which leads to the partitioning and tracking errors when the sizes of targets are different. In order to solve this issue, the GIW-PHD filter was presented in [13]. In this filter, the target kinematic state is assumed to follow the Gaussian distribution, while the extended state is assumed to follow the Wishart distribution. Therefore, the target extended state can be estimated by a RM approach. More discussions of the GIW-PHD filter can be found in [14-17]. However, tracking of closely spaced and maneuvering targets is an unsolved issue of the GIW-PHD filter yet (see section V in [13]), because the partitioning approaches, such as DP-SP, PP, and EMP, fail to provide accurate partitions in such a case. A shape selection partitioning (SSP) algorithm was 
presented in [18] to solve the partitioning problem of closely spaced targets. The SSP employs the target shape to divide measurement set into sub-set, thus it is a promising approach to provide partitions of non-ellipsoidal targets. However, the SSP assumes that the number of closely spaced targets is two, i.e., it cannot apply to the scenario where there are three or more targets closely spaced, and it takes more computation cost compared with other approaches.

This paper proposes a CP approach to improve the performance of the GIW-PHD filter when targets are spaced closely together. The key method of CP is the cooperation between DP-SP and EMP. First, we employ the DP approach to divide the measurement set into cells. Then, we propose an improved detection approach to detect whether a measurement cell ought to be divided into sub-cells. Finally, we improve the EMP algorithm to perform the division. The proposed CP algorithm combines the advantages of the two methods, thus using CP can improve the performance of a GIW-PHD filter than separately using DP-SP and EMP. In addition, with the same performance, the CP takes less computation costs than that of SSP and can handle the cases where the number of targets is larger than two.

The paper is organized as follows. Section 2 introduces the partitioning issues. The CP approach is presented in section 3 . Section 4 shows the simulation results. Conclusions are shown in section 5 .

\section{Measurement Partitioning Issues}

The GIW-PHD filter is a significant implementation of the METT PHD framework, and the details of the GIW-PHD filter can be found in [13]. Measurement partitioning is an integral part of a GIW-PHD filter. Division of the set of measurements, $Z$, into nonempty cells, $W$, is the definition of a partition, $p$. If there is no accurate partition provided by a partitioning approach, the filter will provide inaccurate estimation inevitably.

There are three partitioning approaches used in the GIW-PHD filter, i.e., DP-SP [12], PP and EMP [13], respectively. The DP-SP first uses DP (i.e. using the distance between measurements) to divide the measurement set into cells. Then, the DP-SP will detect whether a cell $W$ contains too many measurements with,

$$
\begin{aligned}
& \hat{N}=\underset{n}{\arg \max } p(|W| \mid N=n), \\
& p\left(|W| \mid N_{j}=n\right)=\text { Pois }(|W|, \gamma n),
\end{aligned}
$$

where $\hat{N}$ denotes the possible number of targets. Pois $(\cdot)$ and $\mid \cdot$ denote the Poisson distribution and the number of elements in a set, respectively. $\gamma$ denotes the expected number measurements generated by a target, and $n$ is a positive integer. If $\hat{N}>1$, it means that $W$ ought to be divided into $\hat{N}$ sub-cells by using K-means++ algorithm [19]. Obviously, formula (1) cannot ensure to calculate a correct $\hat{N}$ because of the randomness of the number of measurements. Moreover, there is no target prediction information used in DP-SP, which means that the performance of the DP-SP approach has no relation with the accuracy of the target prediction information. Hence, the DP-SP approach is insensitive to target maneuvers. However, the K-means++ fail to handle the cases in which targets are of different extensions, i.e., DP-SP can only be used when target extensions are similar.

Compared with DP-SP, the PP and EMP approaches consider the target extensions as important parameters for dividing the measurement set. The PP approach employs the target predicted positions as the clustering centers, and then use the target extensions to assign each measurement to the corresponding center. Therefore, the PP approach is extremely sensitive to target maneuvers. The EMP approach is a generalization of the K-means++ algorithm, which 
incorporates both the cluster extensions and number of measurements in each cluster. The EMP approach is not extremely sensitive to target maneuvers compared with PP, but it will also provide inaccurate partitions if the targets perform maneuvers at a high speed. Hence, the $\mathrm{PP}$ and EMP approaches cannot perform well in the scenario where targets are performing maneuvers.

In order to handle a variety of scenarios, the GIW-PHD filter uses all of these three approaches simultaneously to provide enough partitions. However, if differently sized targets move along a turning track, no partitioning approach can handle such a case. Fig. 1 shows the estimation results of the GIW-PHD filter using the above partitioning approaches when two targets moved along turning tracks. No accurate partitions could be provided by any of the partitioning approaches, thus the target states were estimated incorrectly. In addition to these three approaches, a Kernel-density based partitioning approach was presented in [20]. However, this approach is used in the ET-GM-PHD filter and uses the target prediction information as well as PP and EMP. A fast partitioning approach was presented in [17]. However, only the computation cost of this partitioning approach is lower than that of using the above three approaches, i.e., the corresponding performance of this approach is similar to the above three approaches. The SSP algorithm was presented in [18], which can handle the cases where there are two targets closely spaced. However, the SSP takes more computation cost than other approaches, and the number of closely spaced targets cannot be larger than two. Hence, quickly partitioning of differently sized and closely spaced multi-targets moving along turning tracks is an important issue of METT.

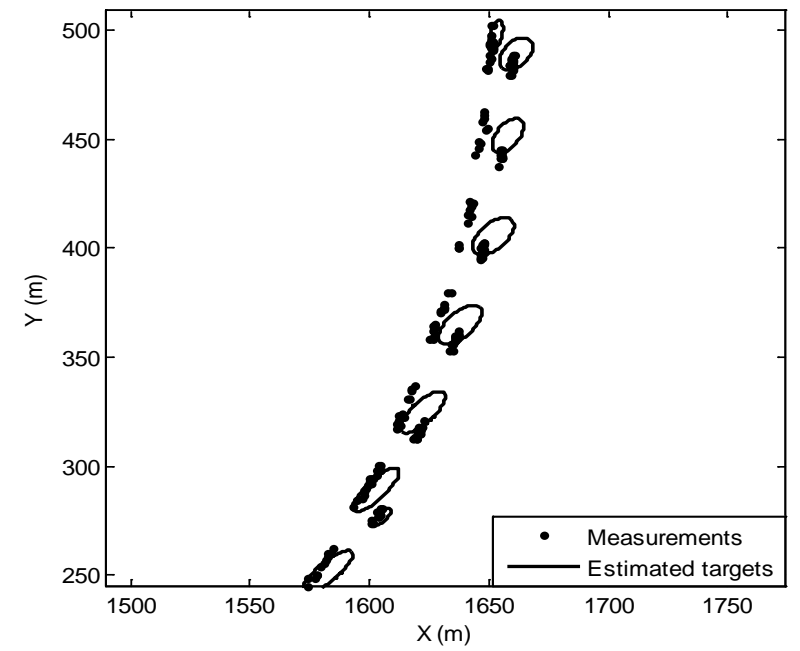

Fig. 1. Estimation results of the GIW-PHD filter of a single trail.

\section{Cooperation Partitioning Algorithm}

The EMP algorithm fails to handle the cases in which the target prediction information is extremely inaccurate, while the DP-SP algorithm cannot divide the measurement cells which are of different sizes. Hence, we combine the DP-SP algorithm with the EMP algorithm to propose the $\mathrm{CP}$ algorithm. The advantage of DP-SP can ensure that the CP will be insensitive to target maneuvers, and the advantage of EMP can make the CP insensitive to the difference of target sizes, compared with using EMP and DP-SP independently. 


\subsection{Definitions and Key Methods of CP}

Suppose $Z_{k}$ denotes the measurement set at time $k$, then the DP algorithm [11] can divide $Z_{k}$ into several measurement cells, i.e., $\left\{W_{k}^{(i)}\right\}$. There are two definitions as follows:

Definition 1: $M_{k \mid k-1}^{(i)}$ is the set of predicted target positions which are close to $W_{k}^{(i)}$, if

$$
\begin{aligned}
& M_{k \mid k-1}^{(i)}=\left\{m_{k \mid k-1}^{(j), d} \mid d_{M}^{(j)}<\vartheta_{\max }\right\}_{j=1}^{J_{k \mid k-1}}, \\
& d_{M}^{(j)}=\sqrt{\left(\bar{z}_{k}^{(i)}-m_{k \mid k-1}^{(j), d}\right)^{T}\left(\bar{z}_{k}^{(i)}-m_{k \mid k-1}^{(j), d}\right)}, \\
& \bar{z}_{k}^{(i)}=\frac{1}{\left|W_{k}^{(i)}\right|} \sum_{z_{k} \in W_{k}^{(i)}} z_{k},
\end{aligned}
$$

where $m_{k \mid k-1}^{(j), d}$ denotes a $d$-dimensional GIW component predicted position at time $k$, and $d$ denotes the dimension of the physical space. $\vartheta_{\max }$ is the maximum distance threshold used in the DP algorithm. $J_{k \mid k-1}$ is the number of the predicted components. $\bar{z}_{k}^{(i)}$ denotes the mean of $W_{k}^{(i)}$.

Definition 2: $\Psi_{k}^{(i)}$ is the set of the means of measurement cells which are close to $W_{k}^{(i)}$, if

$$
\begin{aligned}
& \Psi_{k}^{(i)}=\left\{\bar{z}_{k}^{(\ell)} \mid d_{\Psi}^{(\ell)}<\vartheta_{\max }, \quad \ell \neq i\right\}_{\ell=1}^{N_{W}}, \\
& d_{\Psi}^{(\ell)}=\sqrt{\left(\bar{z}_{k}^{(i)}-\bar{z}_{k}^{(\ell)}\right)^{T}\left(\bar{z}_{k}^{(i)}-\bar{z}_{k}^{(\ell)}\right)},
\end{aligned}
$$

where $\bar{z}_{k}^{(\ell)}$ denotes the mean of a cell $W_{k}^{(\ell)}$. $N_{W}$ denotes the number of the cells divided by DP. In fact, $\Psi_{k}^{(i)}$ denotes the set consisted of the cell means who are around $W_{k}^{(i)}$.

The key method of CP is to use $M_{k \mid k-1}^{(i)}$ and $\Psi_{k}^{(i)}$ to detect whether $W_{k}^{(i)}$ ought to perform a division, and than use an improved EMP to divide $W_{k}^{(i)}$ into sub-cells.

\subsection{Implementation of CP}

Since formula (1) cannot ensure the detection accuracy, we propose a novel approach to detect whether a cell $W_{k}^{(i)}$ ought to perform a division. According to Definition 1 and Definition 2, the CP can detect whether $W_{k}^{(i)}$ ought to be divided into sub-cells with,

$$
\hat{N}=\left\{\begin{array}{cc}
N_{M} & , N_{M}>1 \text { and } N_{\Psi}=0 \\
\underset{n}{\arg \max } \operatorname{Pois}\left(\left|W_{k}^{(i)}\right|, \gamma n\right), & N_{M} \leq 1 \text { and } N_{\Psi}=0, \\
1 & \text { others }
\end{array}\right.
$$

where $N_{M}$ denotes the number of the elements in set $M_{k \mid k-1}^{(i)}$, and $N_{\Psi}$ denotes the number of the elements in set $\Psi_{k}^{(i)}$. The formula (4) means three situations:

(1) If there are other measurement cells around the cell $W_{k}^{(\ell)}$, i.e., $N_{\Psi}>0, W_{k}^{(i)}$ may have been partitioned accurately by DP (i.e. $W_{k}^{(i)}$ is generated by a single target).

(2) In the cases which $N_{\Psi}=0$, if several predicted GIW components are around $W_{k}^{(i)}$, obviously, $W_{k}^{(i)}$ may be generated from $N_{M}$ targets; 
(3) If $N_{\Psi}=0$ and $N_{M} \leq 1$, the prediction information may be inaccurate (such as shown in Fig.

1), thus $\hat{N}$ ought to be estimated by the original approach, i.e., formula (1).

If $\hat{N}>1$ and the situation is (3), the K-means++ approach ought to be used at this time step, because the inaccurate prediction information will affect the performance of EMP. If $\hat{N}>1$ and the situation is (2), $W_{k}^{(i)}$ can be divided into $\hat{N}$ sub-cells by an improved EMP algorithm.

In the original EMP algorithm, the initialized cluster means $\left\{\mu_{t}\right\}_{t=1}^{\hat{N}}$ are defined as the target predicted positions, which leads to the partitioning error when targets are performing maneuvers. Therefore, in the proposed CP algorithm, $\left\{\mu_{t}\right\}_{t=1}^{\hat{N}}$ will be modified by $W_{k}^{(i)}$ to limit the prediction error, by

$$
\begin{aligned}
& \mu_{t}=m_{k \mid k-1}^{(j), d}-\bar{m}_{k \mid k-1}^{(j), d}+\bar{z}_{k}^{(i)}, \\
& \bar{m}_{k \mid k-1}^{(j), d}=\frac{1}{\hat{N}} \sum_{m_{k \mid k-1}^{(j), d} \in M_{k \mid k-1}^{(i)}} m_{k \mid k-1}^{(j), d},
\end{aligned}
$$

where $\bar{m}_{k \mid k-1}^{(j), d}$ denotes the mean of $M_{k \mid k-1}^{(i)}$. Fig. 2 shows the meaning of formula (5). The left part in Fig. 2 shows the scenario where the prediction errors of GIW components are extremely large, and this will lead to the failure of the EMP algorithm. The formula (5), however, can reduce the prediction error, thus the use as a sub-partitioning approach of the EMP algorithm can handle the cases in which the targets are performing maneuvers. Then, other initialized parameters can be given from the corresponding GIW components of $M_{k \mid k-1}^{(i)}$, i.e., the covariances $\sum_{t}=\hat{X}_{k \mid k-1}^{(j)}$ and mixing coefficients $\pi_{t} \propto \gamma^{(j)}$, where $\hat{X}_{k \mid k-1}^{(j)}$ is the estimated extension matrices and $\gamma^{(j)}$ is the estimated numbers of measurements of the $j$ th GIW component. According to [21], the EMP can divide the cell $W_{k}^{(i)}$ into sub-cells as follows:

1) E setp: evaluating the responsibilities using the posterior probability

$$
\omega(c, t)=\frac{\pi_{t} N\left(z_{c} ; \mu_{t}, \Sigma_{t}\right)}{\sum_{\eta=1}^{\hat{N}} \pi_{\eta} N\left(z_{c} ; \mu_{t}, \Sigma_{t}\right)},
$$

where $z_{c}$ is a measurement of cell $W_{k}^{(i)} . \omega(c, t)$ denotes the weight of $z_{c}$ and cluster $t$.

2) M step: re-estimate the parameters using the current responsibilities

$$
\begin{aligned}
& \mu_{t}^{\text {new }}=\frac{1}{N_{t}} \sum_{c=1}^{\left|w_{k}^{(i)}\right|} \omega(c, t) z_{c}, \\
& \sum_{t}^{\text {new }}=\frac{1}{N_{t}} \sum_{c=1}^{\left|W_{k}^{(i)}\right|} \omega(c, t)\left(z_{c}-\mu_{t}^{\text {new }}\right)^{T}\left(z_{c}-\mu_{t}^{\text {new }}\right), \\
& \pi_{t}^{\text {new }}=\frac{N_{t}}{\left|W_{k}^{(i)}\right|}, \\
& N_{t}=\sum_{c=1}^{\left|w_{k}^{(i)}\right|} \omega(c, t),
\end{aligned}
$$

where $N_{t}$ can be interpreted as the effective number of points assigned to cluster $t$. 
3) Evaluate the log likelihood:

$$
\ln \rho(\mu, \Sigma, \pi)=\sum_{c=1}^{\left|W_{k}^{(i)}\right|} \ln \left\{\sum_{k=1}^{\hat{N}} \pi_{t} N\left(z_{c} ; \mu_{t}, \Sigma_{t}\right)\right\} .
$$

If the convergence criterion $\rho$ is not satisfied, return to the E step.

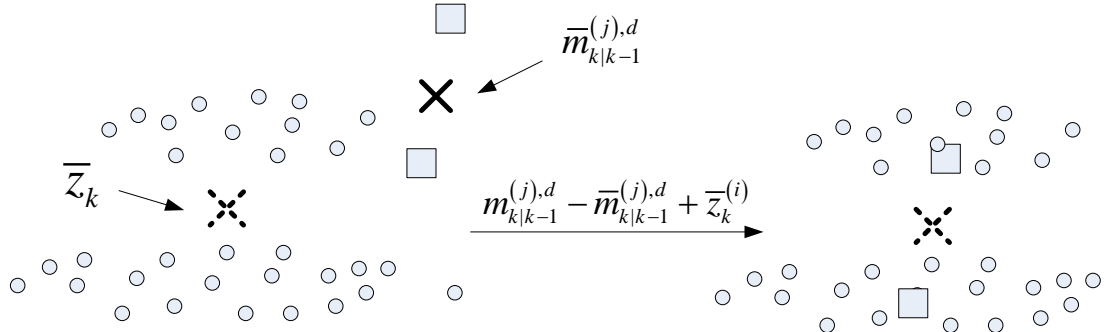

Fig. 2. Illumination of formula (5). The dots denote the measurement of $M_{k \mid k-1}^{(i)}$. The squares denote the predicted positions of GIW components of $M_{k \mid k-1}^{(i)}$.

\section{Simulation results}

The measurement of an extended target is modeled by [5]

$$
\begin{aligned}
& z_{k}^{(j)}=\left(H_{k} \otimes \mathbf{I}_{d}\right) x_{k}^{(i)}+e_{k}^{(j)}, \\
& e_{k}^{(j)}=e_{k, 1}^{(j)}+e_{k, 2}^{(j)},
\end{aligned}
$$

where $\mathbf{I}_{d}$ is a $d$-dimensional unit matrix and $H_{k}=\left[\begin{array}{lll}1 & 0 & 0\end{array}\right] . e_{k, 1}^{(j)}$ is the target extension noise following a Uniform distribution $U\left(A_{k} X_{k} A_{k}^{T}\right)$, and $e_{k, 2}^{(j)}$ is the sensor noise following a Gaussian distribution $\mathcal{N}\left(R_{k}\right)$ (i.e. the measurement model is referred to as $\left.U\left(A_{k} X_{k} A_{k}^{T}\right)+\mathcal{N}\left(R_{k}\right)\right) . X_{k}$ is an extension matrix of the Uniform distribution and $A_{k}$ is a rotation matrix determined by the motion model. $R_{k}$ is a Gaussian measurement noise of the sensor. The number of measurements of each target follows Poisson distribution with the mean $\gamma^{(j)}=15$.

The parameters of simulated scenarios are given as

$$
\begin{aligned}
& T_{s}=1 \mathrm{~s}, \quad p_{D}=0.99, \\
& \mathcal{S}=4000 \times 4000 \mathrm{~m}^{2}, \quad \beta_{F A, k}=6.25 \times 10^{-7}, \\
& R_{k}=\operatorname{diag}([1,1]), \quad Q_{k}=\operatorname{diag}([5,5,0,0]),
\end{aligned}
$$

where $T_{s}$ is the sensor scanning interval and $p_{D}$ is the detection probability. $\mathcal{S}$ denotes the surveillance volume with the rate parameter, i.e. the Poisson mean of clutter measurements is $\mathcal{S} \times \beta_{F A, k}=10$ per scan. $Q_{k}$ and $R_{k}$ are the covariances of process noise and measurement noise respectively.

The parameters of GIW-PHD filter are

$$
\begin{aligned}
w_{0} & =0.1, v_{0}=7, \tau=5, \\
V_{0} & =\operatorname{diag}([50,50]),
\end{aligned}
$$




$$
P_{0}=\operatorname{diag}([25,25,100,100]) \text {, }
$$

where $w_{0}$ is the weight of birth GIW component. $v_{0}, V_{0}$ and $P_{0}$ are inverse Wishart degrees of freedom, inverse scale matrix and Gaussian covariance of birth GIW components, respectively. The distance thresholds of DP-SP is $\vartheta=\{5,10,15, \cdots, 30\}$.

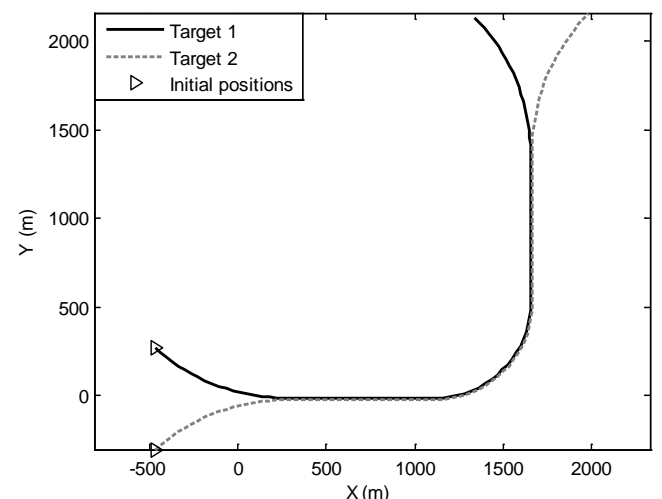

(a)

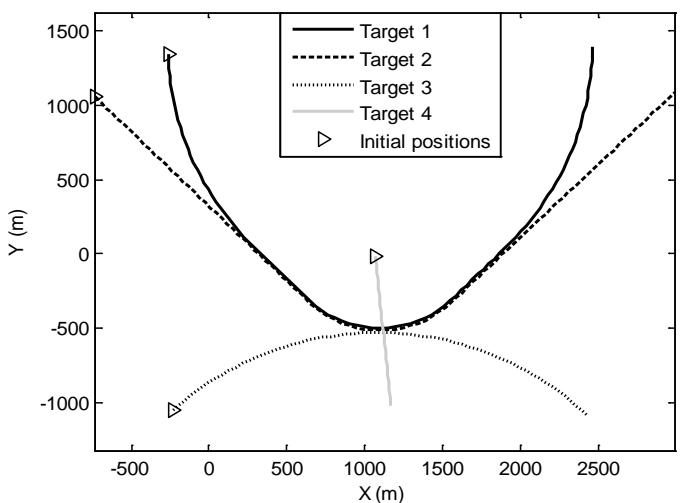

(b)

Fig. 3. True tracks of targets.

\subsection{The Extended Target Optimal Subpattern Assignment Metric (ET-OSPA)}

The performance of a PHD filter is typically evaluated by the optimal subpattern assignment (OSPA) metric [22]. However, the estimated target extensions are not considered by the OSPA metric, thus an ET-OSPA metric [18] was presented for extended target PHD filters with,

$$
\begin{aligned}
& \bar{d}_{p}^{(c)}(X, Y)=\left(\frac { 1 } { n } \left(\min _{\pi \in \prod_{n}} \sum_{i=1}^{m} d^{(c)} \ldots\right.\right. \\
& \left.\left.\times\left(x_{i}, \tilde{X}_{i}, y_{\pi(i)}, \tilde{Y}_{\pi(i)}\right)^{p}+c^{p}(n-m)\right)\right)^{1 / p}, \\
& d^{(c)}\left(x_{i}, \tilde{X}_{i}, y_{\pi(i)}, \tilde{Y}_{\pi(i)}\right)^{p}=\left(x_{i}-y_{\pi(i)}\right)^{p}+R M S E_{\tilde{X}_{i}, \tilde{X}_{\pi(i)}}^{p}, \\
& \operatorname{RMSE}_{\tilde{X}_{i}, \tilde{Y}_{\pi(i)}}^{p}=\left(\operatorname{tr}\left[\left(\tilde{X}_{i}-\tilde{Y}_{\pi(i)}\right)^{2}\right]\right)^{p},
\end{aligned}
$$

where function $R M S E_{\tilde{X}_{i}, \tilde{Y}_{\tilde{R}(i)}}^{p}$ denotes the extension error. $m$ denotes the estimated number of targets and $n$ is the true number of targets. $c$ and $p$ are the given scaling values, respectively. $x_{i}$ and $y_{\pi(i)}$ are the estimated target position and true target position, respectively. $\tilde{X}_{i}$ and $\tilde{Y}_{\pi(i)}$ are estimated and true extension matrix respectively. $\operatorname{tr}[\cdot]$ denotes the trace of a matrix.

\subsection{Turning tracks}

The scenario is simulated by the extended targets with the corresponding extension matrices $X^{(1)}=\operatorname{diag}([20,4])$ and $X^{(2)}=\operatorname{diag}([10,2])$, respectively. They moved closer from time 0-20 s, and then moved linearly together from 21-40 s. They started the turning tracks at time $41 \mathrm{~s}$ and moved linearly together again from 61-80 s. Finally, they moved separately from 81-100 s. Their tracks were shown in Fig. 3 (a). 
Fig. 4 shows the average performances of 100 Monte Carlo runs. Fig. 4 (a) shows the ET-OSPA values of the ET-GM-PHD filter and the GIW-PHD filter using different partitioning approaches. The values of ET-GM-PHD are significantly larger than that of the GIW-PHD filter. The reason of this is that the ET-GM-PHD filter cannot estimate the target extensions (for this filter, the preset extension parameter is given as $\operatorname{diag}([20,20])$ ), thus it fails to handle the cases where target extensions are different. The values of DP-SP are larger than other approaches from 21-40 s and 61-80 s. It means that the partition accuracy of the DP-SP is lower than the CP, SSP and the DP-SP with EMP and PP when targets moved linearly together. However, when targets performed maneuvers form 41-60 s, the performance of the DP-SP with EMP and PP is roughly equal to that of the DP-SP. The reason is that the EMP and PP algorithms are sensitive to target maneuvers, thus the partitions provided by the DP-SP are more accurate. The values of the CP and the values of SSP are roughly equal to that of the DP-SP with EMP and PP from 0-40 s and 61-100 s, but quite lower from 41-60 s. It means that the proposed CP and the SSP can achieve the same performance whit the DP-SP with EMP and PP when targets move linearly together, but the $\mathrm{CP}$ is not sensitive to target maneuvers. The values of the CP are roughly the same as that of the SSP, because the SSP is a maneuver-insensitive partitioning method as well as the CP. However, as shown in Fig. 4 (b), the time cost of the SSP is significantly larger than other approaches, but the time costs of the $\mathrm{CP}$ and the DP-SP with EMP and PP are roughly the same. Therefore, the performances of CP are better than either the SSP or the DP-SP with EMP and PP. Fig. 4 (c) shows the average estimated number of targets. The conclusions of Fig. 4 (a) applies equally to Fig. 4 (c).

Fig. 5 shows the estimation results of the GIW-PHD filter using the CP and using the DP-SP, EMP and PP of a single trial. Fig. 5 (a), (b) and (c) show the results about 21-40 s, 41-60 s and 61-80, respectively. In Fig. 5 (a) and (c), the results of CP and the DP-SP with EMP and PP are the same, because the both methods can handle the cases where targets move uniformly. In Fig. 5 (b), the estimated ellipses of using the DP-SP with EMP and PP do not fit with the extensions of measurements significantly, and sometimes the measurements generated by the two targets are estimated as a single ellipse. It means that the inaccurate partitions provided by the DP-SP with EMP and PP can lead to the estimation error directly. However, in intuitively, the estimation results of using the CP are quite better then using the DP-SP with EMP and PP, and this leads to the results in Fig. 4.

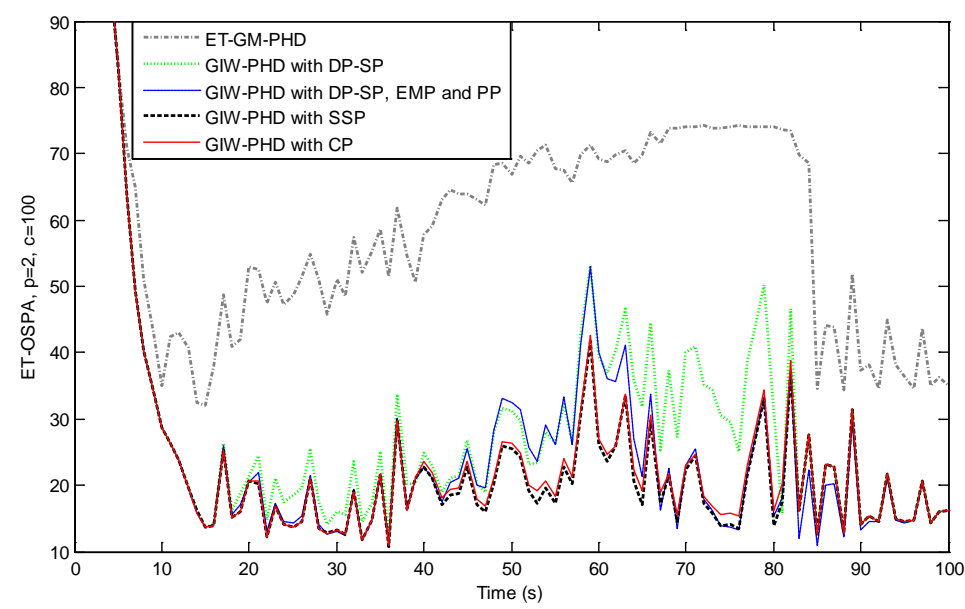

(a) ET-OSPA values. 


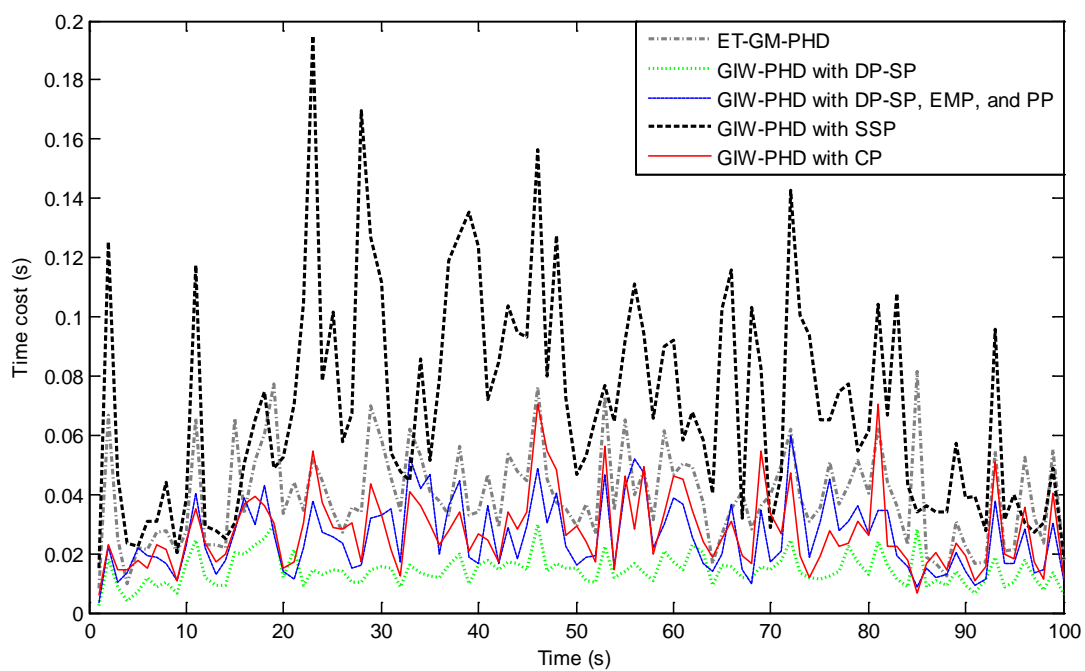

(b) Average time costs.

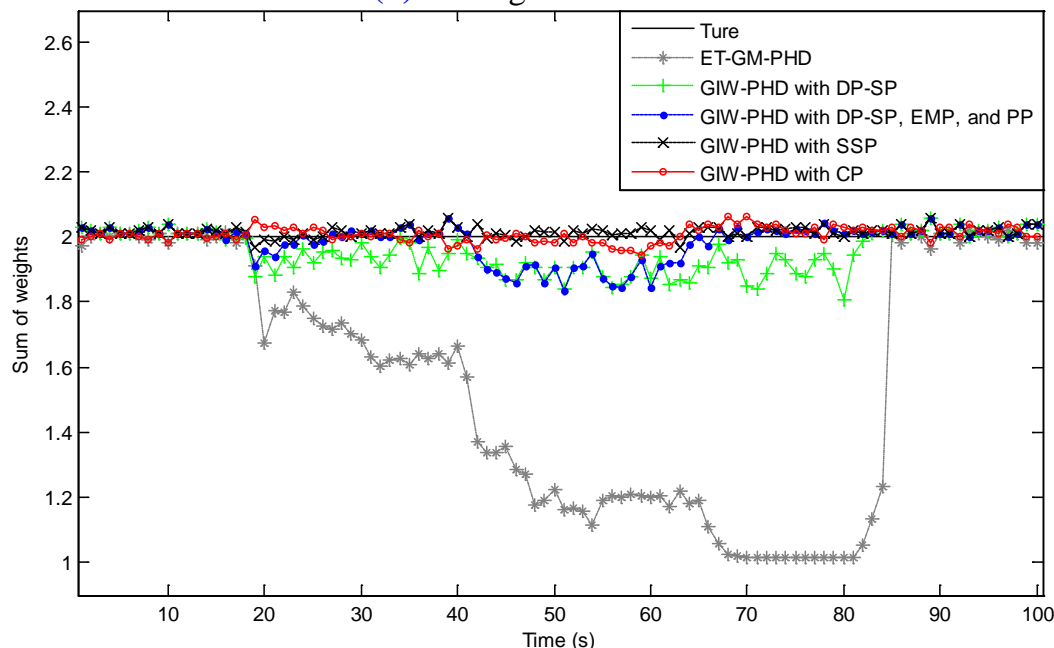

(c) Average estimated number of targets.

Fig. 4. Average performances of 100 runs.

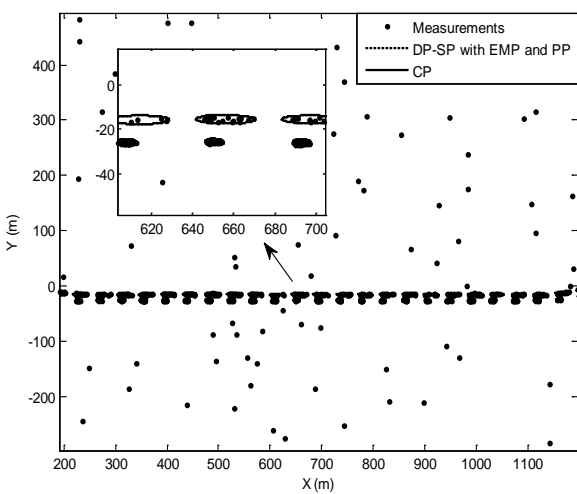

(a)

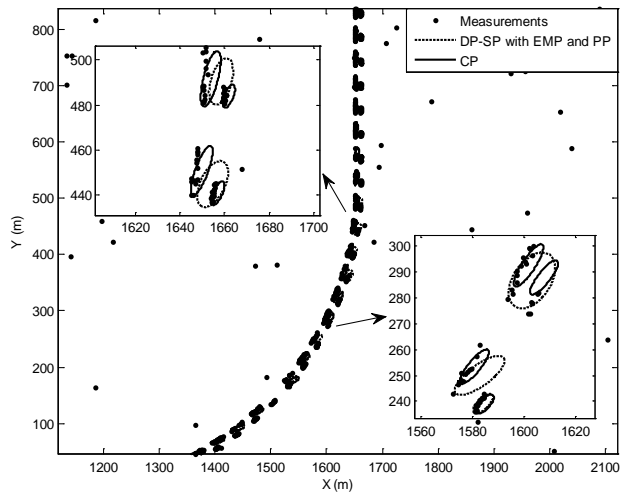

(b) 


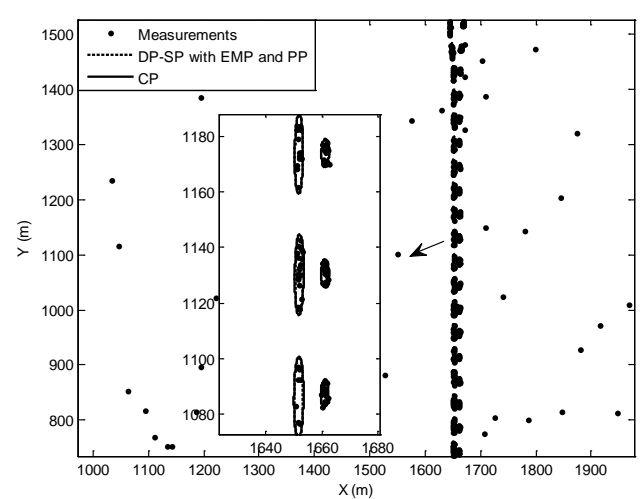

(c)

Fig. 5. Estimation results of a single trail.

\subsection{Crossing tracks}

The scenario is simulated by four extended targets with the corresponding extension matrices $X^{(1)}=\operatorname{diag}([20,4]), X^{(2)}=\operatorname{diag}([10,2]), X^{(3)}=\operatorname{diag}([15,2])$ and $X^{(4)}=\operatorname{diag}([5,1])$, respectively.

Target 1 and target 2 moved closer from 0-30 s, and then moved linearly together from 31-40 s. They moved along the turning tracks from 41-60 s, and then they moved separately. Target 3 generated at $21 \mathrm{~s}$ and moved along a turning track from 21-80 s. Target 4 generated at $41 \mathrm{~s}$ and moved linearly from 41-60 s. All of the targets were closely spaced about time $50 \mathrm{~s}$. Their tracks were shown in Fig. 3 (b). Note that the SSP algorithm fails to handle the cases where the number of closely spaced targets is larger than two, thus the SSP algorithm were not used in this scenario.

Fig. 6 shows the average performances of 100 Monte Carlo runs. Fig. 6 (a) shows the ET-OSPA values of the different approaches. The values of the ET-GM-PHD filter are significantly larger than that of the GIW-PHD filter, which means that the performance of the ET-GM-PHD filter is worse than that of the GIW-PHD filter when there are several targets in the scenario. When targets moved closer, the values of DP-SP, EMP and PP are lower than that of DP-SP. Therefore, the EMP and PP algorithms can improve the performance of the GIW-PHD filter in the scenario where there are several targets closely spaced and crossing. The values of the CP are lower than other approaches from 30-55 s, thus the proposed CP algorithm can achieve better performance than other approaches in this scenario. The same results are shown in Fig. 6 (b), in which the values of the CP are closer to the true values than that of other approaches when targets moved closer.

Fig. 7 shows the estimation results of the GIW-PHD filter using the CP and using the DP-SP, EMP and PP of a single trial. Fig. 7 (a), (b) and (c) show the results from 41-47 s, 47-52 s and 53-59 s, respectively. In Fig. 7 (a), the estimation results of DP-SP, EMP and PP are worse than that of CP because of target maneuvers. In Fig. 7 (b), all of the targets are closely spaced, and the CP performs a better performance than that of the DP-SP, EMP and PP as well. In Fig. 7 (c), the targets move separately. The performances of the both approaches are roughly the same, because the DP-SP can handle the cases where the distance between each target is large enough. The results of Fig. 7 result in the conclusions of Fig. 6. 


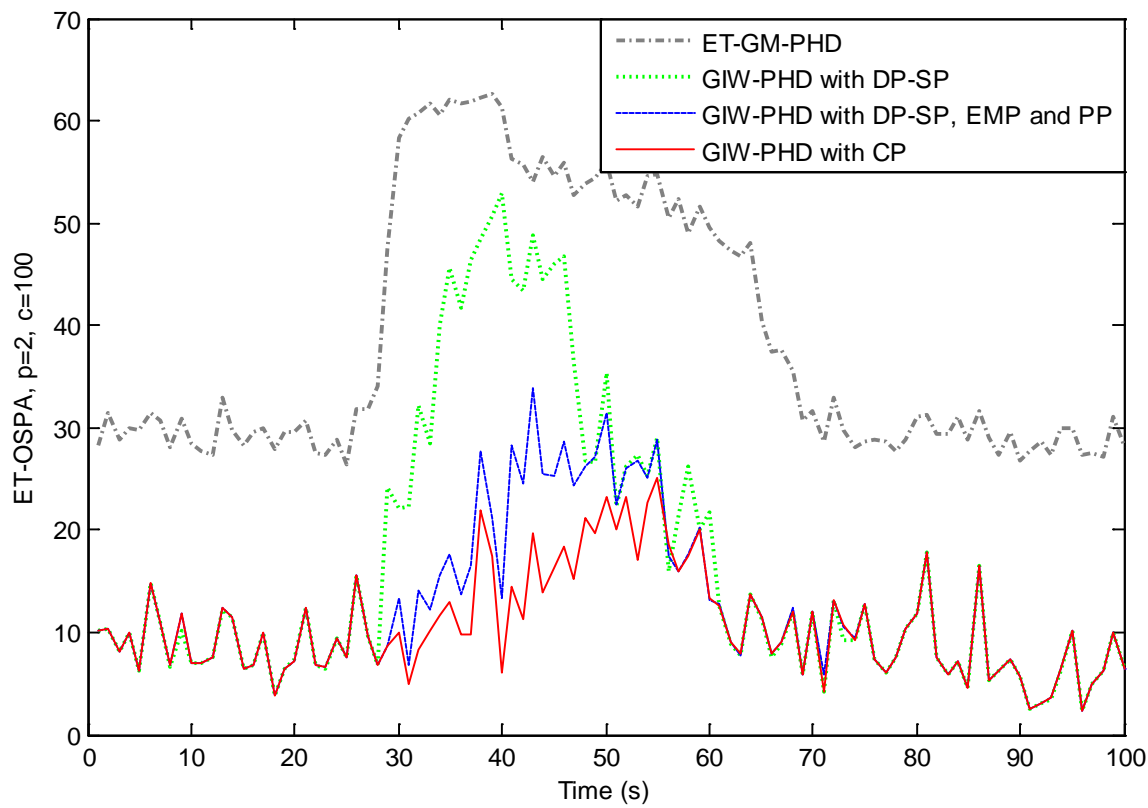

(a) ET-OSPA values.

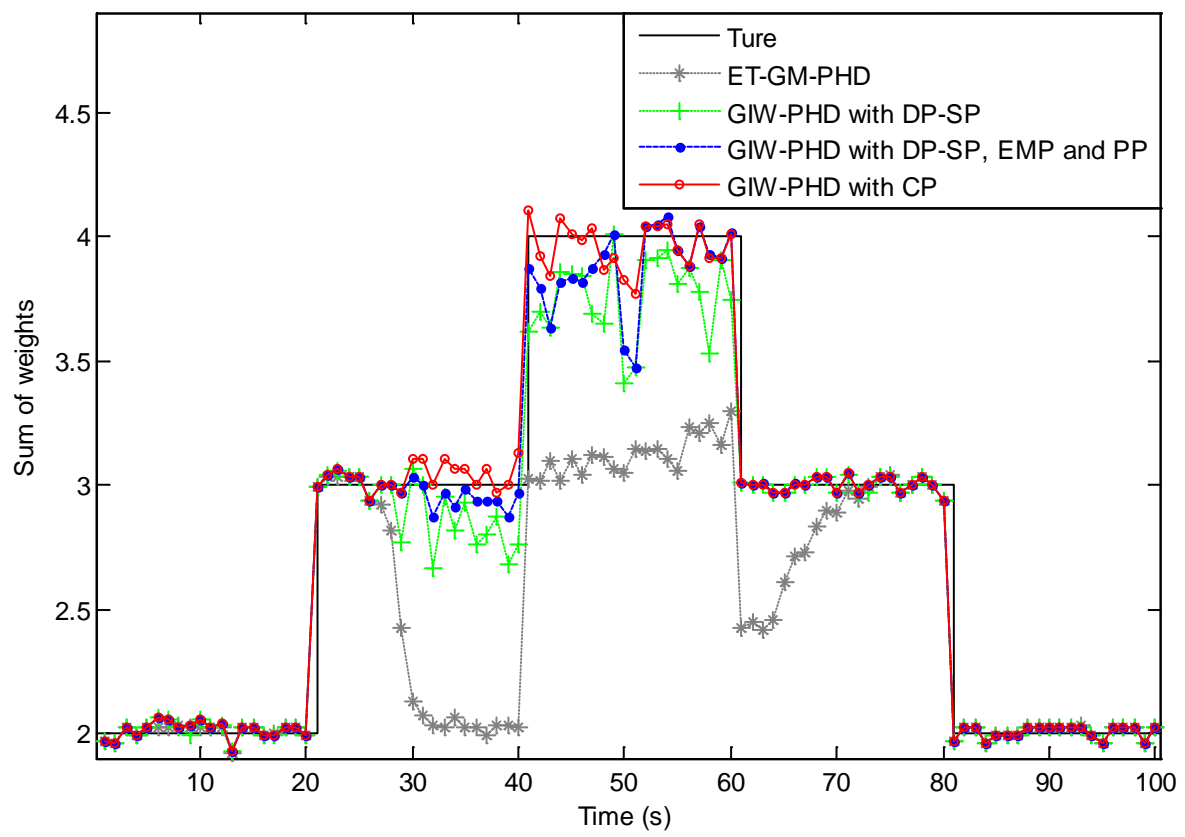

(b) Average estimated number of targets.

Fig. 6. Average performances of 100 runs. 


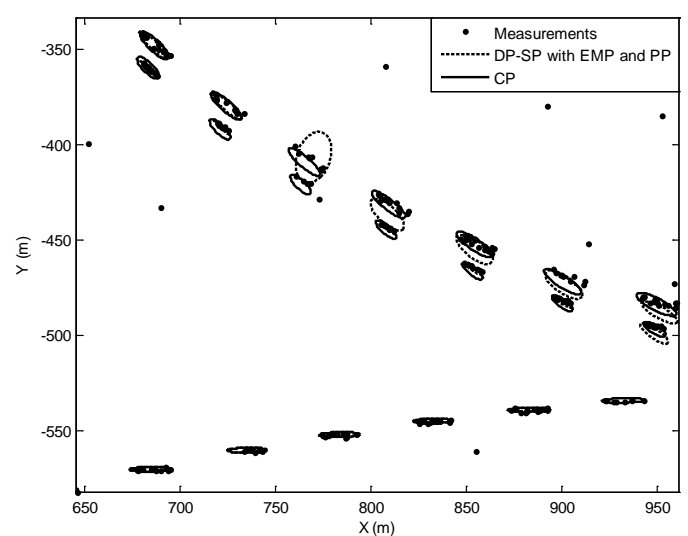

(a)

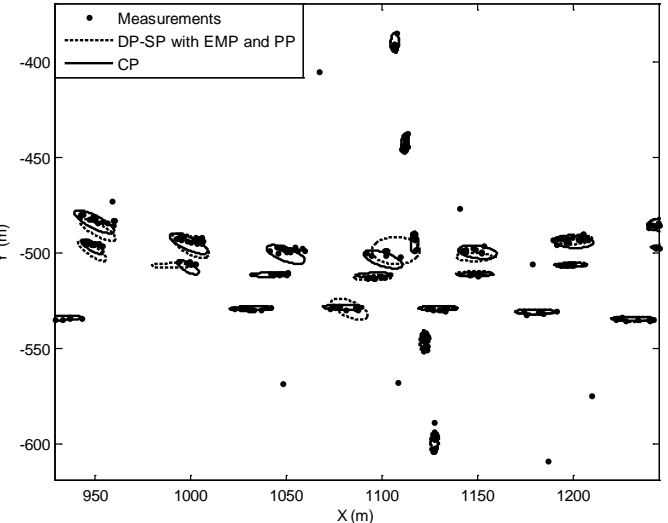

(b)

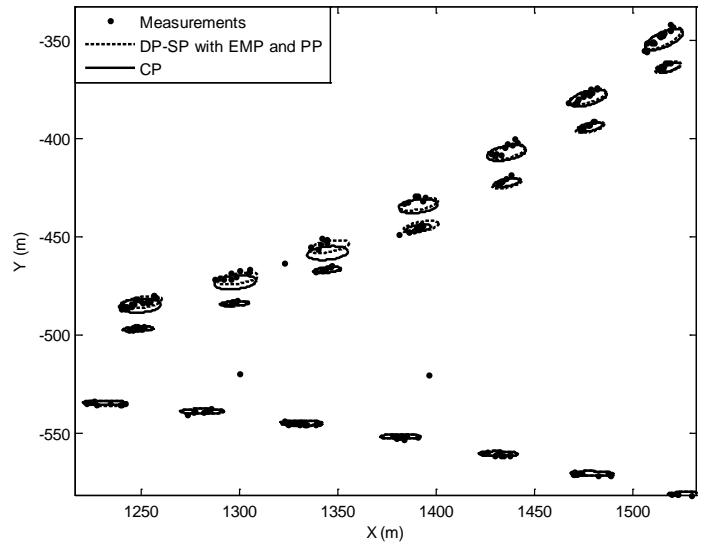

(c)

Fig. 7. Estimation results of a single trail.

\section{Conclusion}

This paper proposes a CP approach to solve the partitioning problem of closely spaced targets. The CP is a combination of DP-SP and EMP, which uses the sub-partition method to improve the initial parameters of the EMP, thus a GIW-PHD filter using CP can achieve better performance than using DP-SP and EMP separately. Compared with the SSP, the CP takes lower time cost than SSP and achieves the same accuracy when the number of targets is two, and the CP can handle the cases where several targets are closely spaced. Therefore, the proposed CP algorithm can achieve better performance than other partitioning approaches used in the GIW-PHD filter.

In the future works, we plan to apply the CP to the particle PHD filters to solve the clustering problem of the particle swarm. When targets are closely spaced, the more accurate clustering results by using the $\mathrm{CP}$ will improve the performance of a particle PHD filter directly.

\section{References}

[1] R. Mahler, "Multi-target Bayes filtering via first-order multi-target moments," IEEE Transactions on Aerospace and Electronic Systems, vol. 39, no. 4, pp. 1152-1178, April, 2003.

Article (CrossRef Link) 
[2] B. N. Vo and W. K. Ma, “The Gaussian Mixture Probability Hypothesis Density Filter," IEEE Transactions on Signal Processing, vol. 54, no. 11, pp. 4091-4104, November, 2006. Article (CrossRef Link)

[3] L. Gao and Y. J. Ma, "Dual Detection-Guided Newborn Target Intensity Based on Probability Hypothesis Density for Multiple Target Tracking,” KSII Transactions on Internet \& Information Systems, vol. 10, no. 10, pp. 5095-5111, October, 2016. Article (CrossRef Link)

[4] W. Koch, "Bayesian approach to extended object and cluster tracking using random matrices," IEEE Transactions on Aerospace and Electronic Systems, vol. 44, no. 3, pp. 1042-1059, July, 2008. Article (CrossRef Link)

[5] M. Feldmann and D. Fränken, "Tracking of Extended Objects and Group Targets Using Random Matrices," IEEE Transactions on Signal Processing, vol. 59, no. 4, pp. 1409-1420, April, 2011. Article (CrossRef Link)

[6] J. Lan, X. R. Li, "Tracking of Maneuvering Non-Ellipsoidal Extended Object or Target Group Using Random Matrix,” IEEE Transactions on Signal Processing, vol. 62, no. 9, pp. 2450-2463, May, 2014. Article (CrossRef Link)

[7] M. Baum and U. D. Hanebeck, "Random hypersurface models for extended object tracking," in Proc. of IEEE International Symposium on Signal Processing and Information Technology, Ajman, United arab emirates, pp.178-183, December, 2009. Article (CrossRef Link)

[8] M. Baum and U. D. Hanebeck, "Shape tracking of extended objects and group targets with star-convex RHMs," in Proc. of 14th International Conference on Information Fusion, Chicago Illinois, USA, pp. 1-8, July, 2011. Article (CrossRef Link)

[9] A. Zea, F. Faion, M. Baum and et al., "Level-set random hypersurface models for tracking non-convex extended objects," in Proc. of 16th International Conference on Information Fusion, Istanbul, Turkey, pp. 1760-1767, July, 2013. Article (CrossRef Link)

[10] R. Mahler, "PHD filters for nonstandard targets, I: Extended targets," in Proc. Of 12th International Conference on Information Fusion, Seattle, WA, USA, pp. 915-921, July, 2009. Article (CrossRef Link)

[11] K. Granström, C. Lundquist and U. Orguner, "A Gaussian mixture PHD filter for extended target tracking," in Proc. of 13th International Conference on Information Fusion, Edinburgh, Scotland, pp. 1-8, July, 2010. Article (CrossRef Link)

[12] K. Granström, C. Lundquist and U. Orguner, "Extended target tracking using a Gaussian-mixture PHD filter," IEEE Transactions on Aerospace and Electronic Systems, vol. 48, no. 4, pp. 3268-3286, October, 2012. Article (CrossRef Link)

[13] K. Granström and U. Orguner, "A PHD filter for tracking multiple extended targets using random matrices," IEEE Transactions on Signal Processing, vol. 60, no. 1, pp. 5657-5671, August, 2012. Article (CrossRef Link)

[14] K. Granström and U. Orguner, "Estimation and maintenance of measurement rates for multiple extended target tracking," in Proc. of 15th Conference on Information Fusion, Singapore, pp. 2170-2176, September, 2012. Article (CrossRef Link)

[15] K. Granström and U. Orguner, "On the reduction of Gaussian inverse Wishart mixtures," in Proc. of 15th Conference on Information Fusion, Singapore, pp. 2162-2169, September, 2012. Article (CrossRef Link)

[16] K. Granstrom, A. Natale, P. Braca and et al, "Gamma Gaussian Inverse Wishart Probability Hypothesis Density for Extended Target Tracking Using X-Band Marine Radar Data," IEEE Transactions on Geoscience \& Remote Sensing, vol. 53, pp. 1-15, July, 2015. Article (CrossRef Link)

[17] Y. Zhang and H. Ji, "A robust and fast partitioning algorithm for extended target tracking using a Gaussian inverse Wishart PHD filter,” Knowledge-Based Systems, vol. 95, pp. 125-141, 2016. Article (CrossRef Link)

[18] P. Li , H. W. Ge, J. L. Yang and et al., "Shape selection partitioning algorithm for Gaussian inverse Wishart probability hypothesis density filter for extended target tracking," IET Signal Processing, vol. 10, no. 9, pp. 1041-1051, October, 2016. Article (CrossRef Link) 
[19] D. Arthur and S. Vassilvitskii, "k-means++: The Advantages of Careful Seeding," in Proc. of ACM-SIAM symposium on Discrete algorithms, Philadelphi, PA, USA, pp. 1027-1035, January, 2007. Article (CrossRef Link)

[20] Y. Han, H. Zhu and C. Z. Han, "Maintaining Track Continuity for Extended Targets Using Gaussian-Mixture Probability Hypothesis Density Filter,” Mathematical Problems in Engineering, no. 3, pp. 1-16, August, 2015. Article (CrossRef Link)

[21] C. M. Bishop, Pattern Recognition and Machine Learning, Springer, New York, 2006. Article (CrossRef Link)

[22] D. Schuhmacher, B. T. Vo and B. N. Vo, "A consistent metric for performance evaluation of multi-object filters," IEEE Transactions on Signal Processing, vol. 56, no. 8, pp. 3447-3457, August, 2008. Article (CrossRef Link)

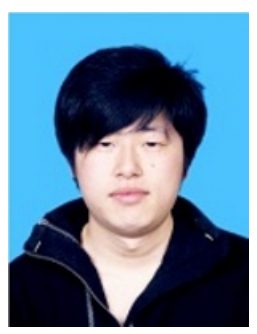

Peng Li was born in 1989. He received his B.S. degree in mathematics and applied mathematics from Langfang Normal University. He is currently a Ph.D. candidate at the School of Internet of Things Engineering in Jiangnan University. His research interests include target tracking and signal processing. E-mail: lipengjiangnan@163.com

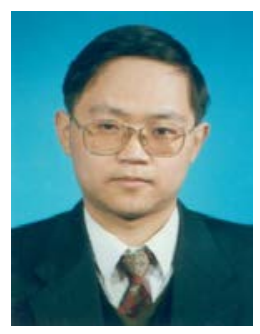

Hongwei Ge was born in 1967. He received the M.S. degree in computer science from Nanjing University of Aeronautics and Astronautics, China, in 1992 and the Ph.D. degree in control engineering from Jiangnan University, China, in 2008. Currently, he is a professor and Ph.D. supervisor in the School of Internet of Things of Jiangnan University. His research interests include target tracking, artificial intelligence, machine learning, pattern recognition and their applications. E-mail: ghw8601@163.com

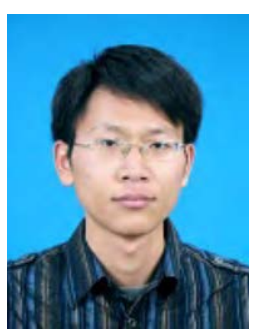

Jinlong Yang was born in 1981. He received his M.S. degree in circuit and system from Northwest Normal University, China in 2009, and his Ph.D. degree in pattern recognition and intelligent system from Xidian University, China in 2012. Currently he is an associate professor in Jiangnan University. His research interests include target tracking, signal processing and pattern recognition. E-mail: yjlgedeng@163.com 